\title{
Performance of Selected Mini Core and Promising Varieties for Productivity and Grain Quality Traits in Rabi Sorghum
}

\author{
Ashwini Karadi ${ }^{1 *}$ and S.T. Kajjidoni ${ }^{2}$ \\ ${ }^{1}$ Indian Institute of Horticultural Research, Banglore, Karnataka, India \\ ${ }^{2}$ University of Agricultural Science, Dharwad, Karnataka, India \\ *Corresponding author
}

\begin{abstract}
A B S T R A C T
\section{Keywords}

Grain Quality

Traits, Rabi

Sorghum

Article Info

Accepted:

16 November 2020

Available Online:

10 December 2020

An investigation was carried out to assess the performance of selected mini core collection and promising varieties of sorghum for grain quality traits and productivity traits. Highly significant difference was observed among the selected mini core accessions and varieties of sorghum for all the traits except true density in selected mini core accessions. Mini core accessions found significantly superior over standard check M35-1 for all the traits except seed yield per plant and true density. IS-15931 was the tallest plant with highest panicle length, seed yield per plant and hundred seed weight with maximum seed size with highest seed protein content. IS-13459 and IS-1041were top performing accessions for seed protein and seed amylose content. Among varieties, SVD-803 was having highest seed yield per plant, hundred seed weight, seed size, seed volume, bulk density and seed amylose content. The top performing superior lines can be used as donar parent in crop improvement programme.
\end{abstract}

\section{Introduction}

Sorghum (Sorghum bicolor (L.) Moench) is an important staple food in several regions of Africa, China and Indian subcontinent particularly in the semi-arid tropics. It is a major food crop in sub-Saharan Africa and South Asia and is the staple food for the most food-insecure people in the world (Bibi et al., 2010).Worldwide, it is cultivated on 41.07 million ha area with production of 58.42 million tonnes in the year approx., 2019-20 (Anonymous, 2019a) In India, sorghum having 5.00 million ha area with 4.5 million tonnes production and $900 \mathrm{~kg} / \mathrm{ha}$ productivity in the year 2019-20 (Anonymous, 2019b). The grain productivity of rabi sorghum in India is lower $(750 \mathrm{~kg} / \mathrm{ha})$ than kharif sorghum (1100 kg/ha) even though rabi sorghum is highly valued because of its excellent grain and fodder quality. The varieties and hybrids developed did not become popular because of poor grain quality and shoot fly susceptibility. In rabi sorghum, breeding for grain quality components like seed size, seed volume, density of seed and nutritional quality traits like amylose and protein content are as important as that of the breeding for higher yield. For many years rabi sorghum growing areas, the roti made 
from Maldandi (M 35-1) is preferred for taste and softness, over the other genotypes. But now days some new genotypes of rabi sorghum are developed which gives better nutritional as well as grain quality than the $M$ 35- 1.

Mini core collection (10\% accessions of the core collection or $1 \%$ of entire collection) represents the whole range of variation of cultivated sorghum and is an ideal material for assessing the exact nature and magnitude of variability of the crop. Mini core collection is considered as a gateway for utilization of diversity present in large germplasm collection for crop improvement (Upadhyaya, et al., 2009). Developing new sorghum genotypes with high yield potential coupled with nutritionally superior quality grains is the prime objective of the breeding programme. The present investigation deals with the details of grain and nutritional quality of mini core collection and sorghum varieties by systematic breeding programme and compared with the local check M35-1.

\section{Materials and Methods}

The plant material for this experiment comprised of (a) 24 selected mini core collection out of 208 mini core accessions based on grain hardness, grain colour and grain luster along with $M$ 35-1 as check variety (Table-1). The mini core collection of sorghum obtained from DSR Hyderabad. (b) Sixteen selected released/promising varieties of rabi sorghum which are commonly grown in northern Karnataka (Table-2). The experiment was laid out in medium deep black soil under rain fed condition was carried out in two experiments at AICRP, UAS, Dharwad during rabi season of 2012-13.The randomized block design was followed separately with two replications and each entry was sown in four rows of $4 \mathrm{~m}$ length with inter row spacing of $45 \mathrm{~cm}$ and intra row spacing of $15 \mathrm{~cm}$. Observations on all quantitative characters like plant height $(\mathrm{cm})$, panicle length $(\mathrm{cm})$, panicle width $(\mathrm{cm})$, seed yield per plot $(\mathrm{g}), 100$ seed weight $(\mathrm{g})$, seed volume $(\mathrm{ml})$, bulk density $(\mathrm{g} / \mathrm{ml})$, true density $(\mathrm{g} / \mathrm{ml})$, seed size $(\mathrm{mm})$, seed protein $(\%)$, seed amylose $(\%)$ and seed yield/plot $(\mathrm{kg})$.

Plant height $(\mathrm{cm})$ : Height of the plant from ground level to the tip of the matured panicle was measured and recorded in centimeters.

Ear head length $(\mathrm{cm})$ : Ear head length was recorded from the base of the panicle to the tip of the panicle and expressed in centimetres.

Ear head width $(\mathrm{cm})$ : Width of the ear head was measured at the broadest point.

100-seed weight $(\mathrm{g})$ : The weight of 100 seeds drawn randomly from randomly selected plants was recorded and expressed in grams.

Grain yield per plant (g): Grains harvested from the plants of each treatment were dried and weighed. The average grain weight of plants is expressed in grams as grain yield per plant.

Seed volume: Countable numbers of seeds were placed in a measuring jar and its volume was noted. Later seed volume per seed was recorded.

Seed volume $(\mathrm{ml})=\frac{\text { Volume }(\mathrm{ml})}{\text { Number of seeds }}$

7. Seed bulk density $(\mathrm{g} / \mathrm{ml})$ : Hundred gram of seeds were weighed and volume was recorded in a measuring jars. It was calculated by using the formula:

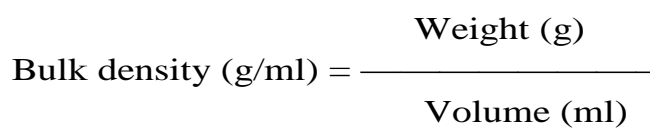


Seed true density: Known weight of seeds placed in a measuring jar containing known quantity of toluene. Increase in volume was recorded after pouring seeds in it. The seed true density was recorded by using the formula:

$$
\text { True density }=\frac{\text { Weight }(\mathrm{g})}{\text { Volume }(\mathrm{ml})}
$$

Seed size (mm): By using Vernier Callipers, length, breadth and thickness of seeds were recorded. Seed size was calculated using the formula.

Seed size $=\sqrt[3]{\text { Length }(\mathrm{mm}) \times \text { breadth }(\mathrm{mm}) \times}$ thickness (mm)

\section{Estimation of biochemical parameters}

Seed protein (per cent): Protein content of selected genotypes was estimated by using Microkjeldhal method. Total nitrogen was estimated by using Kel-plus (digestion and distillation unit). Crude protein value was obtained by multiplying the total nitrogen by the conversion factor.

Amylose content (percent): Total amylose was estimated by following the method of Soubhagya and Bhattacharya (1979), $100 \mathrm{mg}$ sample was taken in a $100 \mathrm{ml}$ volumetric flask, disperse $1 \mathrm{ml}$ of alcohol followed by 10 $\mathrm{ml}$ of $1 \mathrm{~N} \mathrm{NaOH}$ leave it for overnight, make the volume upto $100 \mathrm{ml}$ from distilled water. From this extract $2.5 \mathrm{ml}$ was taken, add $20 \mathrm{ml}$ distilled water, add 3 drops of phenolphthalein indicator, it will change to pink color, add $0.1 \mathrm{~N} \mathrm{HCl}$, till it becomes colourless, now add $1 \mathrm{ml}$ of 0.2 per cent iodine solution, make volume made up to 100 $\mathrm{ml}$. The purple-blue was read at $590 \mathrm{~nm}$.

\section{Statistical analysis}

All treatments were replicated twice and organized in a randomized complete block design (RCBD). Data acquired from the present investigation was subjected to ANOVA (Sheoran et al., 1998). Significance between the treatments were calculated according to Duncan's Multiple Range Test at $\mathrm{p}=0.05$ using GenStat $14^{\text {th }}$ edition.

\section{Results and Discussion}

In the present investigation which included 25 mini core and 16 promising varieties were carried out in order to study the performance varieties of rabi sorghum for eleven quantitative characters. Highly significant difference was observed for all the yield traits like plant height, ear head length and ear head width, hundred seed weight and seed yield per plant in both selected mini core and selected promising varieties. However, highly significant result were observed for all productivity, grain quality and nutritional quality traits like seed volume, seed size, protein and amylose except bulk and true density in selected mini core and bulk density and seed size among the selected promising varieties (Table 3).

This indicates that the selected mini core collection indicating wide genetic variability and there is ample scope for selection of characters from these diverse sources for yield and its components. These findings were in accordance with the findings of Vedansh et al., (2010) and Khandelwal et al., (2016)

\section{Performance of selected mini core and promising varieties for yield, grain and nutritional quality traits in rabi sorghum}

Performance of selected mini core and promising varieties for yield traits in rabi sorghum in table 4 and 5. Selected mini core collection and selected varieties were having wide genetic diversity with tallest to shortest, having longer and wider panicle to shorter panicle. 
Table.1 Selected genotypes of mini core collections of sorghum (Experiment-a)

\begin{tabular}{|c|l|l|l|l|l|}
\hline SI. No. & Accession number & Origin & \multicolumn{1}{|c|}{ Seed hardness } & \multicolumn{1}{|c|}{ Seed lustre } & \multicolumn{1}{|c|}{ Seed color } \\
\hline 1 & IS-473 & USA & Very hard & Lustrous & White \\
\hline 2 & IS-1041 & India & Hard & Lustrous & White \\
\hline 3 & IS-2379 & South Africa & Very hard & Non lustrous & Light brown \\
\hline 4 & IS-3971 & India & Very hard & Intermediate & Creamy straw \\
\hline 5 & IS-4515 & India & Hard & Lustrous & White \\
\hline 7 & IS-5295 & India & Very hard & Intermediate & Chalky white \\
\hline 8 & IS-5301 & India & Very hard & Lustrous & White \\
\hline 9 & IS-12697 & Australia & Hard & Intermediate & Brown \\
\hline 10 & IS-12937 & Ethiopia & Very hard & Lustrous & Light red \\
\hline 11 & IS-12945 & Nicaragua & Very hard & Lustrous & White \\
\hline 12 & IS-13294 & Venezuda & Very hard & Intermediate & Light brown \\
\hline 13 & IS-13459 & Mexico & Hard & Lustrous & Brown \\
\hline 14 & IS-13971 & South Africa & Very hard & Intermediate & Light brown \\
\hline 15 & IS-15931 & Cameroon & Hard & Lustrous & Chalky white \\
\hline 16 & IS-19153 & Sudan & Very hard & Lustrous & White \\
\hline 17 & IS-22720 & Somalia & Hard & Lustrous & White \\
\hline 18 & IS-24139 & Tanzania & Very hard & Intermediate & White \\
\hline 19 & IS-28849 & Yemen & Hard & Intermediate & White \\
\hline 20 & IS-29358 & Lesotho & Very hard & Intermediate & Light yellow \\
\hline 21 & IS-30443 & China & Very hard & Lustrous & Chalky white \\
\hline 22 & IS-30450 & China & Very hard & Non lustrous & Brown \\
\hline 23 & IS-30572 & Cameroon & Very hard & Intermediate & Yellow \\
\hline 24 & IS-13893 & South Africa & Hard & Intermediate & Reddish brown \\
\hline 25 & IS-13782 & South Africa & Very hard & Intermediate & Red \\
\hline & M35-1 & India & Hard & Lustrous & White \\
\hline
\end{tabular}

Table.2 List of selected released/promising varieties of rabi sorghum (Experiment-b)

\begin{tabular}{|c|l|}
\hline SI. No. & \multicolumn{1}{|c|}{ Varieties name } \\
\hline 1 & DSV-4 \\
\hline 2 & SPV-86 \\
\hline 3 & SPV-1829 \\
\hline 4 & BJV-44 \\
\hline 5 & DSV-5 \\
\hline 6 & A-1 \\
\hline 7 & CSV-216R(Phuleyashoda) \\
\hline 8 & PhuleVasudha \\
\hline 9 & PhuleRevathi \\
\hline 10 & M35-1 (Akola source) \\
\hline 11 & M35-1 (Bijapur source) \\
\hline 12 & BarsiJowar \\
\hline 13 & Kodmurki (popular local) \\
\hline 14 & SVD-803(Advanced breeding lines) \\
\hline 15 & SVD-808 (Advanced breeding lines) \\
\hline 16 & SVD-770(Advanced breeding lines) \\
\hline
\end{tabular}


Table.3 Analysis of variance for yield and yield attributing traits in selected mini core collections of sorghum

\begin{tabular}{|c|c|c|c|c|c|c|c|c|c|c|c|}
\hline Parameters & $\begin{array}{c}\text { Plant } \\
\text { height } \\
\text { (cm) }\end{array}$ & $\begin{array}{c}\text { Ear } \\
\text { head } \\
\text { length } \\
(\mathrm{cm})\end{array}$ & $\begin{array}{c}\text { Ear } \\
\text { head } \\
\text { width } \\
(\mathrm{cm})\end{array}$ & $\begin{array}{c}100 \text { seed } \\
\text { weight } \\
(\mathrm{g})\end{array}$ & $\begin{array}{c}\text { Seed } \\
\text { yield/plan } \\
t(g)\end{array}$ & $\begin{array}{c}\text { Seed } \\
\text { volume } \\
(\mathbf{m l})\end{array}$ & $\begin{array}{c}\text { Bulk } \\
\text { density } \\
\text { (g/ml) }\end{array}$ & $\begin{array}{c}\text { True } \\
\text { density } \\
\text { (g/ml) }\end{array}$ & $\begin{array}{l}\text { Seed size } \\
\quad(\mathbf{m m})\end{array}$ & $\begin{array}{c}\text { Protein } \\
\text { content } \\
(\%)\end{array}$ & $\begin{array}{c}\text { Amylose } \\
\text { content } \\
(\%)\end{array}$ \\
\hline \multicolumn{12}{|c|}{ Selected mini core collection of sorghum } \\
\hline P value & $<0.001$ & $<0.001$ & $<0.001$ & $<0.001$ & $<0.001$ & $<0.001$ & 0.036 & 0.799 & $<0.001$ & $<0.001$ & $<0.001$ \\
\hline $\mathbf{V r}$ & 131.63 & 547.77 & 145.9 & 22.78 & 48.36 & 97.01 & 2.11 & 0.71 & 15.17 & 54.31 & 37.93 \\
\hline Mean & 223.84 & 25.58 & 9.06 & 2.85 & 20.09 & 0.04 & 0.96 & 1.24 & 3.42 & 10.64 & 29.64 \\
\hline Range & $\begin{array}{c}163.6- \\
334.2\end{array}$ & $6.4-42.5$ & $\begin{array}{c}4.4- \\
21.50\end{array}$ & $\begin{array}{l}1.97- \\
4.34\end{array}$ & $2.63-44.68$ & $\begin{array}{c}0.02- \\
0.07\end{array}$ & $\begin{array}{c}0.94- \\
0.98\end{array}$ & $\begin{array}{c}1.17- \\
1.34\end{array}$ & $2.79-3.82$ & $\begin{array}{l}7.66- \\
13.78\end{array}$ & $17.5-36.84$ \\
\hline SE \pm & 3.68 & 0.41 & 0.42 & 0.14 & 2.12 & 0 & 0.01 & 0.05 & 0.08 & 0.23 & 1.03 \\
\hline $\begin{array}{l}\text { LSD (P } \\
0.05)\end{array}$ & 10.75 & 1.21 & 1.22 & 0.42 & 6.19 & 0 & 0.02 & 0.16 & 0.24 & 0.68 & 3.01 \\
\hline CV $(\%)$ & 2.3 & 2.3 & 6.5 & 7.1 & 14.9 & 4.5 & 0.9 & 6.2 & 3.4 & 3.1 & 4.9 \\
\hline \multicolumn{12}{|c|}{ Selected varieties of sorghum } \\
\hline P value & $<0.001$ & $<0.001$ & $<0.001$ & $<0.001$ & $<0.001$ & $<0.001$ & 0.0088 & $<0.001$ & 0.009 & $<0.001$ & $<0.001$ \\
\hline $\mathbf{V r}$ & 928.8 & 55.75 & 26.88 & 21.98 & 28.62 & 19.88 & 3.7 & 13.88 & 3.56 & 11.17 & 8.56 \\
\hline \multirow[t]{2}{*}{ Mean } & 251.13 & 19.51 & 6.78 & 3.6 & 32.45 & 0.04 & 0.92 & 1.32 & 3.73 & 9.66 & 23.95 \\
\hline & $\begin{array}{l}191.6- \\
289.5\end{array}$ & $\begin{array}{l}12.2- \\
23.9\end{array}$ & $5.4-6.3$ & $3.15-4.4$ & $10.98-29.2$ & $\begin{array}{c}0.04- \\
0.05\end{array}$ & $\begin{array}{c}0.71- \\
0.98\end{array}$ & $\begin{array}{l}1.12- \\
1.54\end{array}$ & $3.4-4.1$ & $8.09-8.86$ & $\begin{array}{l}20.85- \\
26.67\end{array}$ \\
\hline SE \pm & 0.84 & 0.39 & 0.13 & 0.08 & 1.69 & 0 & 0.08 & 0.02 & 0.06 & 0.23 & 0.7 \\
\hline $\begin{array}{l}\text { LSD (P } \\
0.05)\end{array}$ & 2.53 & 1.17 & 0.4 & 0.23 & 5.08 & 0 & 0.23 & 0.07 & 0.18 & 0.69 & 2.12 \\
\hline CV (\%) & 0.5 & 2.8 & 2.8 & 3 & 7.3 & 3.5 & 2.9 & 3.7 & 6.4 & 3.3 & 4.1 \\
\hline
\end{tabular}


Table.4 Mean performances of selected mini core collection of sorghum for yield, grain and nutritional quality traits

\begin{tabular}{|c|c|c|c|c|c|c|c|c|c|c|c|}
\hline Parameters & $\begin{array}{c}\text { Plant } \\
\text { Height } \\
\text { (cm) }\end{array}$ & $\begin{array}{c}\text { Ear head } \\
\text { length }(\mathrm{cm})\end{array}$ & $\begin{array}{c}\text { Ear head } \\
\text { width }(\mathrm{cm})\end{array}$ & $\begin{array}{c}\text { Hundred } \\
\text { seed weight } \\
\text { (g) }\end{array}$ & $\begin{array}{c}\text { Seed yield } \\
\text { per plant } \\
(\mathrm{g})\end{array}$ & $\begin{array}{c}\text { Seed } \\
\text { volume } \\
(\mathrm{ml})\end{array}$ & $\begin{array}{c}\text { Bulk } \\
\text { density }(\mathrm{g} / \mathrm{ml})\end{array}$ & $\begin{array}{c}\text { True } \\
\text { density }(\mathrm{g} / \mathrm{ml})\end{array}$ & $\begin{array}{l}\text { Seed size } \\
\quad(\mathrm{mm})\end{array}$ & Protein (\%) & $\begin{array}{c}\text { Amylose } \\
(\%)\end{array}$ \\
\hline IS-473 & $214.00 \mathrm{efg}$ & $38.30 \mathrm{k}$ & $21.50 \mathrm{k}$ & 2.65 cde & $5.91 \mathrm{abc}$ & 0.03 cdef & 0.96 bcde & $1.34 \mathrm{a}$ & $2.93 \mathrm{a}$ & $8.09 \mathrm{ab}$ & $31.67 \mathrm{f}$ \\
\hline IS-1041 & $193.50 \mathrm{c}$ & $22.50 \mathrm{f}$ & $7.70 \mathrm{f}$ & $3.45 \mathrm{fg}$ & $25.78 \mathrm{hi}$ & $0.05 \mathrm{~h}$ & $0.95 \mathrm{ab}$ & $1.17 \mathrm{a}$ & $3.61 \mathrm{ef}$ & $12.47 \mathrm{i}$ & $34.17 \mathrm{fg}$ \\
\hline IS-2379 & $215.50 \mathrm{efg}$ & $31.10 \mathrm{ij}$ & $14.00 \mathrm{i}$ & 2.62 bcde & $7.03 \mathrm{abc}$ & $0.03 \mathrm{cdef}$ & 0.95 abcde & $1.24 \mathrm{a}$ & $3.01 \mathrm{ab}$ & $10.28 \mathrm{fg}$ & $31.67 \mathrm{f}$ \\
\hline IS-3971 & $165.10 \mathrm{a}$ & $9.80 \mathrm{~b}$ & $5.10 \mathrm{ab}$ & 2.61 bcde & $5.71 \mathrm{abc}$ & $0.03 \mathrm{def}$ & 0.96 bcde & $1.24 \mathrm{a}$ & $3.04 \mathrm{ab}$ & $11.16 \mathrm{~h}$ & $34.17 \mathrm{fg}$ \\
\hline IS-4515 & $243.50 \mathrm{jlm}$ & $18.30 \mathrm{de}$ & 7.30 ef & $3.94 \mathrm{hij}$ & $44.68 \mathrm{k}$ & $0.06 \mathrm{k}$ & 0.96 bcde & $1.20 \mathrm{a}$ & $3.73 \mathrm{f}$ & 9.86 ef & $35.84 \mathrm{~g}$ \\
\hline IS-5295 & 210.00 def & $31.70 \mathrm{ij}$ & $11.60 \mathrm{gh}$ & $1.97 \mathrm{a}$ & $5.48 \mathrm{abc}$ & $0.02 \mathrm{a}$ & $0.97 \mathrm{de}$ & $1.27 \mathrm{a}$ & $2.79 \mathrm{a}$ & $8.53 \mathrm{bc}$ & $35.84 \mathrm{~g}$ \\
\hline IS-5301 & $236.00 \mathrm{ijkl}$ & $12.20 \mathrm{c}$ & $5.80 \mathrm{bcd}$ & $3.51 \mathrm{fgh}$ & $34.30 \mathrm{j}$ & $0.05 \mathrm{~h}$ & 0.97 cde & $1.27 \mathrm{a}$ & $3.68 \mathrm{f}$ & $9.41 \mathrm{de}$ & $35.84 \mathrm{~g}$ \\
\hline IS-12697 & $221.00 \mathrm{fgh}$ & $27.30 \mathrm{~h}$ & $10.90 \mathrm{~g}$ & 2.50 bcde & $3.21 \mathrm{a}$ & $0.03 \mathrm{def}$ & 0.96 bcde & $1.34 \mathrm{a}$ & $3.05 \mathrm{ab}$ & $8.09 \mathrm{ab}$ & $34.01 \mathrm{fg}$ \\
\hline IS-12937 & $225.00 \mathrm{ghi}$ & $22.40 \mathrm{f}$ & $6.60 \mathrm{cdef}$ & 2.46 bcde & $31.83 \mathrm{ij}$ & 0.03 def & 0.97 cde & $1.26 \mathrm{a}$ & $3.28 \mathrm{bcd}$ & $8.97 \mathrm{~cd}$ & $31.67 \mathrm{f}$ \\
\hline S-12945 & 204.50 cde & $25.30 \mathrm{~g}$ & $6.90 \mathrm{def}$ & $2.75 \mathrm{e}$ & $11.82 \mathrm{cdef}$ & $0.03 \mathrm{fg}$ & $0.97 \mathrm{de}$ & $1.24 \mathrm{a}$ & 3.59 ef & $12.03 \mathrm{i}$ & $31.67 \mathrm{f}$ \\
\hline IS-13294 & $259.50 \mathrm{n}$ & $30.70 \mathrm{ij}$ & $7.70 \mathrm{f}$ & $2.16 \mathrm{ab}$ & $3.77 \mathrm{ab}$ & $0.03 \mathrm{bcd}$ & 0.96 bcde & $1.21 \mathrm{a}$ & 3.54 ef & $7.66 \mathrm{a}$ & $35.84 \mathrm{~g}$ \\
\hline IS-13459 & $270.50 \mathrm{o}$ & $21.20 \mathrm{f}$ & $7.10 \mathrm{def}$ & $16.88 \mathrm{a}$ & 10.73 bcde & $0.03 a b$ & $0.95 \mathrm{abcd}$ & $1.23 \mathrm{a}$ & $2.90 \mathrm{a}$ & $13.78 \mathrm{j}$ & $34.17 \mathrm{fg}$ \\
\hline IS-13971 & $253.20 \mathrm{mn}$ & $30.40 \mathrm{i}$ & 6.50 bcdef & 2.52 bcde & $16.03 \mathrm{defg}$ & 0.03 efg & 0.96 abcde & $1.19 \mathrm{a}$ & 3.37 cde & $9.41 \mathrm{de}$ & $34.17 \mathrm{fg}$ \\
\hline IS-15931 & $303.50 \mathrm{p}$ & 40.001 & $12.80 \mathrm{hi}$ & $4.11 \mathrm{ij}$ & $33.58 \mathrm{j}$ & $0.06 \mathrm{k}$ & 0.96 bcde & $1.25 \mathrm{a}$ & $3.75 \mathrm{f}$ & $12.47 \mathrm{i}$ & $33.34 \mathrm{fg}$ \\
\hline S-19153 & $167.20 \mathrm{a}$ & $17.90 \mathrm{~d}$ & 6.00 bcde & $3.36 \mathrm{fg}$ & $31.65 \mathrm{ij}$ & $0.05 \mathrm{hi}$ & 0.95 abcde & $1.24 \mathrm{a}$ & $3.71 \mathrm{f}$ & $10.50 \mathrm{fgh}$ & $25.84 \mathrm{e}$ \\
\hline IS-22720 & 204.90 cde & $6.40 \mathrm{a}$ & $4.40 \mathrm{a}$ & 3.80 ghi & $17.54 \mathrm{efg}$ & $0.05 \mathrm{ij}$ & 0.95 abcde & $1.21 \mathrm{a}$ & $3.68 \mathrm{f}$ & $11.16 \mathrm{~h}$ & $20.83 \mathrm{~b}$ \\
\hline IS-24139 & $334.20 \mathrm{q}$ & 40.801 & $11.00 \mathrm{~g}$ & $2.26 \mathrm{abcd}$ & 12.44 cdef & $0.03 \mathrm{bc}$ & $0.97 \mathrm{cde}$ & $1.24 \mathrm{a}$ & 3.54 cef & $12.03 \mathrm{i}$ & $20.83 \mathrm{~b}$ \\
\hline IS-28849 & $193.00 \mathrm{c}$ & $17.50 \mathrm{~d}$ & $5.40 \mathrm{abc}$ & $4.34 \mathrm{j}$ & 9.20 abcd & 0.071 & 0.96 bcde & $1.24 \mathrm{a}$ & $3.67 \mathrm{f}$ & $10.72 \mathrm{gh}$ & $17.50 \mathrm{a}$ \\
\hline IS-29358 & $180.70 \mathrm{~b}$ & $22.00 \mathrm{f}$ & $5.70 \mathrm{abcd}$ & $2.74 \mathrm{de}$ & $20.70 \mathrm{gh}$ & $0.04 \mathrm{~g}$ & $0.97 \mathrm{cde}$ & $1.27 \mathrm{a}$ & 3.60 ef & $10.28 \mathrm{fg}$ & $20.84 \mathrm{bc}$ \\
\hline IS-30443 & $277.50 \mathrm{o}$ & $42.50 \mathrm{~m}$ & $20.80 \mathrm{k}$ & 2.53 bcde & $28.52 \mathrm{ij}$ & $0.03 \mathrm{def}$ & 0.96 abcde & $1.27 \mathrm{a}$ & $3.00 \mathrm{a}$ & $12.03 \mathrm{i}$ & 24.17 bde \\
\hline IS-30450 & $198.50 \mathrm{~cd}$ & $19.40 \mathrm{e}$ & $5.40 \mathrm{abc}$ & 2.31 abcde & $2.63 \mathrm{a}$ & 0.03 cdef & $0.95 \mathrm{abc}$ & $1.18 \mathrm{a}$ & $3.28 \mathrm{bc}$ & $13.78 \mathrm{j}$ & $22.50 \mathrm{bcd}$ \\
\hline IS-30572 & $197.50 \mathrm{c}$ & $30.70 \mathrm{ij}$ & $19.30 \mathrm{j}$ & $2.20 \mathrm{abc}$ & $18.00 \mathrm{fg}$ & 0.03 bcde & $0.94 \mathrm{a}$ & $1.22 \mathrm{a}$ & $3.68 \mathrm{f}$ & $12.36 \mathrm{i}$ & 20.84 bc \\
\hline IS-13893 & $232.00 \mathrm{hij}$ & $31.80 \mathrm{j}$ & $5.30 \mathrm{abc}$ & 2.57 bcde & $28.15 \mathrm{ij}$ & $0.03 \mathrm{cdef}$ & 0.96 bcde & $1.26 \mathrm{a}$ & 3.55 ef & $10.28 \mathrm{fg}$ & $34.17 \mathrm{fg}$ \\
\hline IS-13782 & $163.60 \mathrm{a}$ & $31.90 \mathrm{j}$ & $5.80 \mathrm{bcd}$ & 2.63 bcde & $33.45 \mathrm{j}$ & $0.03 \mathrm{fg}$ & 0.97 bcde & $1.22 \mathrm{a}$ & $3.69 \mathrm{f}$ & $11.16 \mathrm{~h}$ & $36.84 \mathrm{~g}$ \\
\hline M35-1 & 232.00hijk & $17.40 \mathrm{~d}$ & 6.00 bcde & $3.30 \mathrm{f}$ & 60.191 & $0.05 \mathrm{jk}$ & $0.98 \mathrm{e}$ & $1.34 \mathrm{a}$ & $3.82 \mathrm{f}$ & $9.41 \mathrm{de}$ & $22.50 \mathrm{bcd}$ \\
\hline
\end{tabular}


Table.5 Mean performances of selected released varieties of sorghum for grain quality and biochemical characters

\begin{tabular}{|c|c|c|c|c|c|c|c|c|c|c|c|}
\hline Parameters & $\begin{array}{c}\text { Plant height } \\
\text { (cm) }\end{array}$ & $\begin{array}{l}\text { Ear head } \\
\text { length } \\
\text { (cm) }\end{array}$ & $\begin{array}{c}\text { Ear head } \\
\text { width } \\
(\mathrm{cm})\end{array}$ & $\begin{array}{c}\text { Hundred } \\
\text { seed weight } \\
\text { (g) }\end{array}$ & $\begin{array}{c}\text { Seed yield } \\
\text { per plant } \\
\text { (g) }\end{array}$ & $\begin{array}{c}\text { Seed } \\
\text { volume }\end{array}$ & Seed size & $\begin{array}{c}\text { Bulk } \\
\text { density }\end{array}$ & True density & Protien & Amylose \\
\hline DSV-4 & $253.00 \mathrm{~g}$ & $23.50 \mathrm{i}$ & 6.90 cde & $4.05 \mathrm{~g}$ & $37.64 \mathrm{c}$ & $0.05 \mathrm{def}$ & $3.63 \mathrm{abc}$ & $0.94 \mathrm{c}$ & $1.55 \mathrm{e}$ & 9.84 def & $20.85 \mathrm{a}$ \\
\hline SPV-86 & $191.60 \mathrm{a}$ & $20.90 \mathrm{fgh}$ & $6.30 \mathrm{~b}$ & $3.38 \mathrm{abc}$ & $29.00 \mathrm{~b}$ & $0.04 \mathrm{~b}$ & $3.82 \mathrm{bc}$ & $0.78 \mathrm{a}$ & $1.34 \mathrm{bcd}$ & $8.09 \mathrm{a}$ & $20.85 \mathrm{a}$ \\
\hline SPV-1829 & $280.50 \mathrm{i}$ & 19.80 ef & $6.30 \mathrm{~b}$ & $3.21 \mathrm{a}$ & $28.04 \mathrm{~b}$ & 0.05 cde & $3.71 \mathrm{bc}$ & $0.72 \mathrm{a}$ & $1.21 \mathrm{ab}$ & $10.55 \mathrm{fg}$ & $20.85 \mathrm{a}$ \\
\hline BJV-44 & $271.00 \mathrm{~h}$ & $21.00 \mathrm{fgh}$ & $7.10 \mathrm{def}$ & $3.49 \mathrm{bc}$ & $30.99 \mathrm{~b}$ & 0.05 cde & $3.71 \mathrm{bc}$ & $0.98 \mathrm{c}$ & $1.50 \mathrm{de}$ & $9.84 \mathrm{def}$ & $22.50 \mathrm{ab}$ \\
\hline DSV-5 & $271.00 \mathrm{~h}$ & $20.60 \mathrm{fg}$ & $5.40 \mathrm{a}$ & $3.48 \mathrm{bc}$ & $10.98 \mathrm{a}$ & $0.05 \mathrm{bc}$ & $3.40 \mathrm{a}$ & $0.86 \mathrm{~b}$ & $1.13 \mathrm{a}$ & 9.84 def & $24.17 \mathrm{bcd}$ \\
\hline A-1 & $249.00 \mathrm{f}$ & $12.20 \mathrm{a}$ & $6.25 \mathrm{~b}$ & $3.19 \mathrm{a}$ & $42.61 \mathrm{c}$ & $0.04 \mathrm{a}$ & $3.67 \mathrm{bc}$ & $0.96 \mathrm{c}$ & $1.25 \mathrm{abc}$ & $10.28 \mathrm{ef}$ & $24.19 \mathrm{bcd}$ \\
\hline Phule Yashoda & $289.50 \mathrm{j}$ & $21.80 \mathrm{gh}$ & $7.15 \mathrm{ef}$ & $3.22 \mathrm{a}$ & $26.85 \mathrm{~b}$ & $0.05 \mathrm{bcd}$ & $3.68 \mathrm{bc}$ & $0.97 \mathrm{c}$ & 1.40 bcde & $10.06 \mathrm{def}$ & $22.50 \mathrm{ab}$ \\
\hline Phule Vasudha & $279.00 \mathrm{i}$ & $23.90 \mathrm{i}$ & $8.45 \mathrm{~h}$ & $3.15 \mathrm{a}$ & $31.38 \mathrm{~b}$ & 0.05 cde & $3.70 \mathrm{bc}$ & $0.97 \mathrm{c}$ & $1.24 \mathrm{abc}$ & $11.15 \mathrm{~g}$ & $25.84 \mathrm{de}$ \\
\hline Phule Revathi & $270.50 \mathrm{~h}$ & $22.00 \mathrm{~h}$ & $7.35 \mathrm{fg}$ & $3.32 \mathrm{ab}$ & $40.35 \mathrm{c}$ & $0.05 \mathrm{bcd}$ & $3.68 \mathrm{bc}$ & $0.98 \mathrm{c}$ & $1.31 \mathrm{abcd}$ & $9.40 \mathrm{~cd}$ & $22.50 \mathrm{ab}$ \\
\hline M35-1 (Akola) & $249.50 \mathrm{f}$ & $18.50 \mathrm{~cd}$ & $6.70 \mathrm{bcd}$ & $3.57 \mathrm{bcd}$ & $26.31 b$ & $0.05 \mathrm{gh}$ & $3.71 \mathrm{bc}$ & $0.74 \mathrm{a}$ & $1.34 \mathrm{bcd}$ & $9.41 \mathrm{~cd}$ & $23.34 \mathrm{bc}$ \\
\hline M35-1 (Bijapur) & $224.50 \mathrm{~b}$ & $17.70 \mathrm{c}$ & $6.60 \mathrm{bc}$ & $3.85 \mathrm{efg}$ & $42.21 \mathrm{c}$ & 0.05 cdef & $3.58 \mathrm{ab}$ & $0.98 \mathrm{c}$ & 1.44 cde & $9.63 \mathrm{de}$ & $25.84 \mathrm{de}$ \\
\hline Barsi Jowar & $230.50 \mathrm{c}$ & 18.70 cde & $6.50 \mathrm{bc}$ & $4.40 \mathrm{~h}$ & $28.07 \mathrm{~b}$ & $0.06 \mathrm{i}$ & $3.77 \mathrm{bc}$ & $0.96 \mathrm{c}$ & $1.25 \mathrm{abc}$ & $9.40 \mathrm{~cd}$ & $25.84 \mathrm{de}$ \\
\hline Kodmurki & $240.50 \mathrm{~d}$ & $16.10 \mathrm{~b}$ & $6.30 \mathrm{~b}$ & $3.61 \mathrm{cde}$ & $29.20 \mathrm{~b}$ & 0.05 efg & $3.90 \mathrm{~cd}$ & $0.94 \mathrm{c}$ & $1.22 \mathrm{ab}$ & $8.86 \mathrm{bc}$ & $26.67 \mathrm{e}$ \\
\hline SVD-803 & $247.00 \mathrm{ef}$ & $17.90 \mathrm{~cd}$ & $6.65 \mathrm{bc}$ & $3.96 \mathrm{fg}$ & $49.68 \mathrm{~d}$ & $0.06 \mathrm{~h}$ & $3.84 \mathrm{bc}$ & $0.95 \mathrm{c}$ & $1.32 \mathrm{abcd}$ & $9.40 \mathrm{~cd}$ & $25.84 \mathrm{de}$ \\
\hline SVD-808 & $245.50 \mathrm{e}$ & $18.50 \mathrm{~cd}$ & $6.85 \mathrm{cde}$ & $3.78 \mathrm{def}$ & $28.46 \mathrm{~b}$ & $0.05 \mathrm{fgh}$ & $4.09 \mathrm{~d}$ & $0.95 \mathrm{c}$ & 1.40 bcde & $8.53 \mathrm{ab}$ & $25.86 \mathrm{de}$ \\
\hline SVD-770 & $225.50 \mathrm{~b}$ & $19.10 \mathrm{de}$ & $7.60 \mathrm{~g}$ & $3.90 \mathrm{fg}$ & $37.38 \mathrm{c}$ & $0.05 \mathrm{efg}$ & $3.74 \mathrm{bc}$ & $0.97 \mathrm{c}$ & $1.26 \mathrm{abc}$ & $10.28 \mathrm{ef}$ & $25.55 \mathrm{cde}$ \\
\hline
\end{tabular}


Table.6 Top five superior performing genotypes identified for seed yield and grain quality traits in selected mini core collections of sorghum

\begin{tabular}{|c|c|c|c|c|c|c|c|c|}
\hline \multirow[t]{2}{*}{ Traits } & Plant height & Panicle & Panicle width & $\begin{array}{c}\text { Seed yield } \\
\text { per plant }\end{array}$ & $\begin{array}{c}100 \text { seed } \\
\text { weight }\end{array}$ & \multirow[t]{2}{*}{$\begin{array}{l}\text { Seed size } \\
\quad(\mathbf{m m})\end{array}$} & \multirow[t]{2}{*}{ Protein (\%) } & \multirow{2}{*}{$\begin{array}{c}\text { Amylose } \\
(\%)\end{array}$} \\
\hline & $(\mathrm{cm})$ & length $(\mathrm{cm})$ & $(\mathrm{cm})$ & (g) & (g) & & & \\
\hline \multirow[t]{5}{*}{1} & IS-24139 & IS-30443 & IS-473 & IS-4515 & IS-28849 & IS-15931 & & IS-4515 \\
\hline & $(334.20)$ & $(42.50)$ & $(21.5)$ & $(44.68)$ & (4.34) & $(3.75)$ & IS-13459 & IS-5295 \\
\hline & & & & & & & IS-30450 & IS-5301 \\
\hline & & & & & & & (13.78) & IS-13294 \\
\hline & & & & & & & & $(35.84)$ \\
\hline \multirow[t]{8}{*}{2} & IS-15931 & IS-24139 & IS-30443 & IS-15931 & IS-15931 & IS-4515 & IS-1041 & IS-1041 \\
\hline & $(303.50)$ & $(40.80)$ & $(20.80)$ & $(33.58)$ & $(4.11)$ & $(3.73)$ & IS-15931 & IS-3971 \\
\hline & & & & & & & $(12.47)$ & IS-13459 \\
\hline & & & & & & & & IS-13971 \\
\hline & & & & & & & & IS-13893 \\
\hline & & & & & & & & $(34.17)$ \\
\hline & IS-30443 & IS-15931 & IS-30572 & IS-13782 & IS-4515 & S-19153 & IS-30572 & IS-12697 \\
\hline & $(277.50)$ & $(40.00)$ & $(19.30)$ & $(33.45)$ & $(3.94)$ & (3.71) & $(12.36)$ & $(34.01)$ \\
\hline Mean & 223.84 & 25.58 & 9.06 & 20.09 & 2.85 & 3.42 & 10.64 & 29.64 \\
\hline C.D. at $5 \%$ & 10.75 & 1.21 & 1.22 & 0.28 & 0.43 & 0.24 & 0.68 & 3.01 \\
\hline C.D. at $1 \%$ & 18.25 & 4.09 & 1.26 & 0.38 & 0.58 & 0.39 & 0.93 & 4.15 \\
\hline
\end{tabular}


Table.7 Top five superior performing genotypes identified for seed yield and grain quality traits in selected promising varieties of sorghum

\begin{tabular}{|c|c|c|c|c|c|c|c|c|}
\hline SI. No. & Plant height $(\mathrm{cm})$ & $\begin{array}{c}\text { Ear head } \\
\text { length }(\mathrm{cm})\end{array}$ & $\begin{array}{c}\text { Ear head } \\
\text { width }(\mathrm{cm})\end{array}$ & $\begin{array}{c}\text { Seed yield/plant } \\
\text { (g) }\end{array}$ & $\begin{array}{c}100 \text { seed } \\
\text { weight }(g)\end{array}$ & Seed & Seed Protein & $\begin{array}{c}\text { Seed } \\
\text { Amylose }\end{array}$ \\
\hline & & & & & & Size $(\mathrm{mm})$ & $(\%)$ & $(\%)$ \\
\hline \multirow[t]{3}{*}{1} & Phule Yashoda & Phule & SVD-770 & SVD-803 & Barsi Jowar & SVD-808 & Phule & Kodmurki \\
\hline & & Vasudha & & & & & Vasudha & \\
\hline & $(289.50)$ & (23.9) & (7.6) & $(49.68)$ & $(4.4)$ & $(4.09)$ & (11.15) & (26.67) \\
\hline \multirow[t]{3}{*}{2} & SPV-1829 & DSV- 4 & Phule & A-1 & DSV-4 & Kodmurki & SPV-1829 & SVD-808 \\
\hline & & & Revathi & & & & & \\
\hline & $(280.50)$ & $(23.5)$ & $(7.35)$ & $(42.61)$ & $(4.05)$ & (3.9) & $(10.55)$ & $(25.86)$ \\
\hline \multirow[t]{3}{*}{3} & Phule Vasudha & Phule Revathi & BJV-44 & M35-1 (Bijapur) & SVD-803 & SVD-803 & A-1 & SVD-803 \\
\hline & & & & & & & & Barsi Jowar \\
\hline & $(279.00)$ & $(22)$ & (7.1) & $(42.21)$ & $(3.96)$ & $(3.84)$ & $(10.28)$ & $(25.84)$ \\
\hline Mean & 251.91 & 19.61 & 6.78 & 32.45 & 3.60 & 3.73 & 9.66 & 23.95 \\
\hline $\begin{array}{c}\text { C.D. at } \\
5 \%\end{array}$ & 2.53 & 1.17 & 0.4 & 0.55 & 0.23 & 0.18 & 0.69 & 2.12 \\
\hline $\begin{array}{c}\text { C.D. at } \\
1 \%\end{array}$ & 9.39 & 2.01 & 1.4 & 0.76 & 1.01 & 0.32 & 0.95 & 2.93 \\
\hline
\end{tabular}


The seed yield per plant and hundred seed weight having wide range variability compared to standard commercial check M35-1. The selected mini core and selected varieties were having huge variation for seed volume, bulk density and seed size. These materials were good source of nutritional quality traits like seed protein and seed amylose

\section{Plant height (cm)}

Highly significant difference was observed among the mini core accessions for plant height. Significant superior accessions were found for the plant height compared to standard check M35-1 presented in Table 3. The tallest plant observed was IS-24139 $(334.2 \mathrm{~cm})$ and shortest plant observed was IS-13782 $(163.6 \mathrm{~cm})$. The average plant height was $223.84 \mathrm{~cm}$ in selected mini core accessions. Among selected promising varieties, highly significant difference was observed among varieties. The tallest plant recorded was phule yashoda $(289.50 \mathrm{~cm})$ and shortest plant was observed as SPV-86 $(191.60 \mathrm{~cm})$. The average plant height noticed that $251.13 \mathrm{~cm}$ in Table 4 .

\section{Ear head length (cm)}

For ear head length, highly significant difference was observed among the mini core accessions. Significant superior accessions were found for ear head length over standard check M35-1. The longest ear head length observed was IS-30443 $(42.5 \mathrm{~cm})$ and shortest ear head length recorded was IS$22720(6.4 \mathrm{~cm})$. The average ear head length was $25.58 \mathrm{~cm}$.Wheras, among released varieties, highly significant difference was observed for ear head length. Variety A1 $(12.2 \mathrm{~cm})$ was recorded as longest and Phule vasudha $(23.9 \mathrm{~cm})$ was observed as shortest for ear head length. Mean of the ear head length was $19.15 \mathrm{~cm}$.

\section{Ear head width (cm)}

Significant superior accessions were found for ear head length over standard check M35-1 and highly significant difference was observed among the selected mini core accessions for ear head width. The widest ear head width observed was IS-473 $(21.50 \mathrm{~cm})$ and narrowest ear head width recorded was IS-22720 (4.4cm). Average ear head width was $9.06 \mathrm{~cm}$. Among varieties also highly significant difference was observed whereas, the maximum ear head width recorded in Phule vasudha $(8.45 \mathrm{~cm})$ and minimum was DSV $-5(5.4 \mathrm{~cm})$. The average ear head width in varieties was $6.78 \mathrm{~cm}$.

\section{Hundred seed weight (gm)}

Highly significant difference was observed among the mini core accessions for hundred seed weight. Significant superior accessions were found for the hundred seed weight compared to standard check M35-1(3.30gm) such as IS-4515 (3.94gm), IS-15931(4.11gm), IS-22720 (3.80gm) and IS-28849 (4.34gm). The maximum hundred seed weight was observed was IS-28849 (4.34gm) and minimum noticed was IS-5295 (1.97gm). The mean of the hundred seed weight was $2.85 \mathrm{gm}$. Among selected varieties, highly significant difference was observed among varieties but some of the varieties were non-significant or on par with each other which was shown in the table 4. The maximum hundred seed weight was observed was Barsi Jowar (4.4gm) and minimum was recorded in phule vasudha $(3.15 \mathrm{gm})$. The average hundred seed weight was $3.6 \mathrm{gm}$.

\section{Seed yield per plant (gm)}

Among the selected mini core accessions, highly significant difference was observed for seed yield per plant. None of the accessions found as significant superior compared to standard check M35-1 (60.19gm). The highest 
seed yield per plant was observed in IS-4515 $(44.68 \mathrm{gm})$ and lowest yield was recorded in IS$30450(2.63 \mathrm{gm})$. The average seed yield per plant was $20.09 \mathrm{gm}$. In case of selected varieties, highly significant difference was noticed. The highest seed yield per plant was observed was SVD-803 (49.68gm) and lowest was DSV-5 $(10.98 \mathrm{gm})$.The mean of the seed yield per plant was $32.45 \mathrm{gm}$. Most of the varieties are on par with each other for seed yield.

\section{Seed volume $(\mathrm{ml})$}

In the present study, highly significant difference was observed for seed volume. IS$28849(0.07 \mathrm{ml})$ was recorded for maximum seed volume and significantly superior than standard check M35-1(0.054 ml). The lowest seed volume was observed in IS-5295 $(0.02 \mathrm{ml})$ and average was $0.04 \mathrm{ml}$.Whereas, highly significant difference was noticed among varieties and highest observed was Barsi Jowar $(0.06 \mathrm{ml})$ and SVD-803 $(0.06 \mathrm{ml})$. The lowest was A-1 (0.03 ml) and mean was $0.04 \mathrm{ml}$.

\section{Seed size $(\mathbf{m m})$}

Most of the accessions were on par with each other with M35-1 and also shown highly significant difference was observed among the accessions. The average seed size was $3.42 \mathrm{~mm}$. Among varieties, significant superior SVD-808 $(4.09 \mathrm{~mm})$ recorded for maximum seed size and lowest was DSV-5 $(3.97 \mathrm{~mm})$. The average seed size was $3.73 \mathrm{~mm}$.

\section{Bulk density (g/ml)}

None of the accession found as significant superior than M35-1 but they highly significant difference was found for bulk density. Mean of the bulk density was $0.96 \mathrm{~g} / \mathrm{ml}$. Most of the varieties were on par with each other. Varieties were significantly different for bulk density and average bulk density was $0.92 \mathrm{~g} / \mathrm{ml}$.

\section{True density $(\mathrm{g} / \mathrm{ml})$}

All the accessions were on par with each other and also with standard check M35-1 recorded in selected mini core collections. The average value was observed was $1.24 \mathrm{~g} / \mathrm{ml}$. Among selected varieties, highly significant difference was found for this trait and mean value was recorded was $1.32 \mathrm{~g} / \mathrm{ml}$.

\section{Seed protein $(\%)$}

Highly significant difference was found among the selected mini core accessions. Most of the accessions were found as significant superior than M35-1 (9.41\%). Average seed protein content was recorded was $10.64 \%$ in selected mini core accessions. Among selected varieties, highly significant difference was found for this trait.

The highest seed protein content was recorded was in Phule Vasudha (11.15\%) and found as significantly superior than M35-1. The lowest seed protein was recorded was SPV-86 (80.9\%). The mean value was recorded was $9.66 \%$. Most of the varieties were on par with each other having same seed protein content.

\section{Seed amylose $(\%)$}

Most of the accessions were found as significantly superior for seed amylose content compare to M35-1(22.5\%).Highly significant difference was found among selected mini core accessions and several accessions were on par with each other for seed amylose content.

The accession with highest seed amylose content was recorded was IS-13782 (36.84\%) which was on par with most of the accessions. The lowest seed amylose content was recorded was IS-28849 (17.50\%) and average seed amylose content was $29.64 \%$. Whereas, among selected varieties highly significant difference was observed for seed amylose. Kodmurki was having highest seed amylose content $26.67 \%$ with lowest was $20.85 \%$. DSV-4, SPV-86 and SPV-1829 were on par with each other having seed amylose content $(20.85 \%)$.

The average seed amylose content was $23.95 \%$ in the selected varieties. Most of the varieties 
were on par with each other having same seed amylose content.

Mean performance of top five superior selected mini core accessions were identified for seed yield and grain quality traits in rabi sorghum (Table 6 and 7). IS 15931 was the tallest plant with highest panicle length, seed yield per plant and hundred seed weight with maximum seed size with highest seed protein content.IS-15931 which is hard, lustrous and chalky white which can be used as donor parent in breeding programme. IS-13459 and IS-1041 were top performing accessions for seed protein and seed amylose content. IS-1041 which is hard, lustrous and white in colour which is having superior grain and nutritional quality.

Among varieties, SVD-803 was recorded highest for seed yield per plant, hundred seed weight, seed size, seed volume, bulk density and seed amylose content. Most of the varieties are superior more than one trait which was presented in Table

\section{Acknowledgement}

The senior author is greatful to DSR Hyderabad for providing mini core collection.

\section{References}

Anonymous. World Agricultural production, 2019a, 21.

Anonymous. World Agricultural production, 2019b, 21

Bibi, A., Sadaqat, H. A., Akram, H. M. and Mohammed, M. I. 2010. Physiological markers for screening sorghum (Sorghum bicolor) germplasm under stress condition." International Journal of Agriculture and Biological Sciences. 12(3): 451-455.

Khandelwal, V., Shukla, M., Jodha, B.S., Nathawat, V.S. and Dashora, S.K. 2016. Genetic parameters and character association in sorghum (Sorghum bicolor (L.) Moench). Indian Journal of Science and Technology. 8(22):73-90.

Mudjisihino, R. and Damardjati, D.S. Prospects usefulness sorghum as a source of food and feed. Journal of Agricultural Research Development. 1987; 1:1-5.

Sowbhagya CM, Bhattacharya KR. Simplified determination of amylose in milled rice starch. Staerke, 1979; 31:159-163.

Upadhyaya HD, Gowda CLL, Reddy KN, Singh S. Augmenting the pearl millet [Pennisetum glaucum (L.) R. Br.)] Core collection for enhancing germplasm utilization in crop improvement. Crop Science. 2009; 49: 573-580.

Vedansh, Singh SK, Kerkhi SA, Singh A, Kumar M, Kumar V. Variability, Heritability and genetic advance for forage yield and quality traits in forage sorghum [Sorghum bicolor (L.) Moench]. Progressive Agriculture 2010; 10(2):400401.

\section{How to cite this article:}

Ashwini Karadi and Kajjidoni, S.T. 2020. Performance of Selected Mini Core and Promising Varieties for Productivity and Grain Quality Traits in Rabi Sorghum. Int.J.Curr.Microbiol.App.Sci. 9(12): 2357-2368. doi: https://doi.org/10.20546/ijcmas.2020.912.280 